\title{
Nutrition Risk Classification: A Reproducible and Valid Tool for Nurses
}

\author{
Debra S. Kovacevich, RN, MPH, CNSN*†; Anthony R. Boney, RN, BSN, CNSN*; \\ Carol L. Braunschweig, PhD, RD, CNSD ; Anne Perez, RN, BSN, CNSN*; \\ and MaRY Stevens, RN, BSN* \\ *Parenteral and Enteral Nutrition Team, University of Michigan Medical Center, Ann Arbor; †College of Pharmacy, University \\ of Michigan, Ann Arbor, and $\ddagger$ Department of Human Nutrition and Dietetics, University of Illinois at Chicago
}

\begin{abstract}
Background:Incorporating the nursing staff to assist with the screening process on admission will allow patients who are at nutritional risk to be assessed by registered dietitians earlier in their hospital stay. The goal of this study was to develop an objective, valid, reproducible nutrition screen for use by registered nurses (RNs) to allow for nutrition classifications of hospitalized patients. Methods: The current nursing admission assessment form was modified to contain questions on weight loss history, percentage of ideal body weight, and alterations in dietary intake and gastrointestinal function. Assessments were completed within 48 hours of admission. On the basis of the answers to these questions, patients were classified as "at nutritional risk" or "low nutritional risk." In phase 1 , to assess reproducibility of the form, a prospective study between staff $\mathrm{RNs}$ and a nutritionist was undertaken on 186 consecutive adult admissions. Nutrition screening and classification was done independently by both practitioners. In phase 2 of the study, prospective validation of the form contrasting prealbumin (PAB) levels with $R N$ nutritional risk classification ( $n=56)$ was investigated. Results: Interobserver agreement of nutrition classification between $\mathrm{RN}$ and nutritionist was $97.3 \%(p=.95)$. Twenty-nine patients were classified at low nutritional risk (27 normal PAB and 2 low $P A B) ; 27$ patients were classified as at nutritional risk (16 normal $\mathrm{PAB}$ and 11 low $\mathrm{PAB})\left(\chi^{2}=8.9, p<\right.$ .003 , power 0.8 ). The sensitivity of the tool was $84.6 \%$. Conclusion: To our knowledge, this is the first nutrition screening tool designed for staff $\mathrm{RNs}$ that has been tested for both validity and reproducibility.
\end{abstract}

Correspondence: Debra S. Kovacevich, Parenteral and Enteral Nutrition Team, Department of Pharmacy Services, UH/B2D301/Box 0008, 1500 E. Medical Center Drive, Ann Arbor, MI 48109-008.

0884-5336/87/1201-0020\$03.00/0

Nutrition in Clinical Practice 12:20-25, February 1997

Copyright 1997 American Society for Parenteral and Enteral Nutrition
The prevalence of malnutrition in hospitalized patients has been estimated to be as high as $48 \% .^{1,2}$ Malnutrition has been associated with increased morbidity and mortality, increased length of hospital stay, and increased cost. ${ }^{3.4}$ Recently, in an attempt to rectify this situation, the Joint Commission on Accreditation for Hospital Organizations (JCAHO) mandated that nutrition screens be completed for all hospitalized patients in a timely fashion. ${ }^{5}$ This mandate challenges professionals responsible for the nutrition care of hospitalized patients. The existing staff in typical nutrition departments are insufficient to provide timely screens for all patients. ${ }^{6}$ In the current financial climate of medical reform it is unlikely that hospital administration will allocate additional funding to accomplish this task. The challenge to health care professionals becomes how to accomplish timely, reproducible, and valid nutrition screens with existing hospital personnel.

Several methods have been developed to screen and assess hospitalized patients to identify those individuals who may be or are at risk for malnutrition. ${ }^{7-14}$ Yet few of these nutrition screening and assessment tools except for the subjective global assessment or SGA have been tested for reproducibility (the ability of a test to produce consistent results when repeated under the same conditions from person to person) and validity (the tool measures what it is supposed to measure) by a variety of health care professionals. The SGA was developed by Baker and colleagues ${ }^{15,18}$ and emphasizes clinical assessment of nutritional status by stressing historical, symptomatic, and physical parameters. The tool has also been shown to be reproducible in patients undergoing major gastrointestinal surgery, in patients admitted to a medical-surgical gastroenterology service, in renal dialysis patients, and in liver transplant candidates. ${ }^{17-20}$

A significant majority of the questions and physical assessment techniques required in the SGA are 
completed by registered nurses upon admission and recorded in the nursing assessment forms. Incorporation of an objective, valid, and reproducible nutrition screen within the standard nursing admission form meets the JCAHO mandate. This technique also allows professionals with formal training in nutrition to optimize their efficiency in prioritizing patients for indepth nutrition assessments.

The purpose of this study was to modify the existing nursing admission assessment form to include reproducible and valid nutrition information for nurses to identify patients at nutritional risk.

\section{MATERIALS AND METHODS}

Phase 1: Testing the Reproducibility of the Modified Nursing Assessment Form. A. Modification of the nursing assessment form. The current nursing assessment tool was modified to contain information on four categories: (1) diagnosis, (2) nutrition intake history, (3) ideal body weight standards, and (4) weight history. Within each of these categories, criteria were added relating to nutritional status (Table 1). The criteria were arranged according to the amount of time required for completion by nurses. When the patient answered yes to any of the criteria, the nurse classified the patient "at nutritional risk" and was not required to complete further categories. If the patient did not meet any of the criteria in any of the categories, the patient was considered to be at "low nutritional risk."

Critical diagnoses that categorized patients at nutritional risk included the following: anorexia nervosa/bulimia nervosa, malabsorption (celiac sprue, ulcerative colitis, Crohn's disease, and short bowel syndrome), multiple trauma (closed head injuries, multiple fractures, and gun shot wounds), major gastrointestinal surgery within the past year, cachexia (temporal wasting and muscle wasting), coma, diabetes, end-stage liver disease, end-stage renal disease, nonhealing wounds, and decubitus ulcers. Nutrition intake history included information on the presence of vomiting for $>5$ days, diarrhea of $>500 \mathrm{~mL}$ for 2 days, and a reduced oral intake of $<1 / 2$ of normal for $>5$ days. If the patient exhibited or recalled any of these criteria, the patient was classified as at nutritional risk. Ideal body weight standards were evaluated by comparing the patient's current weight-for-height to their ideal body weight. If the patient was $<80 \%$ of their ideal body weight, the patient was considered to be at nutritional risk. A chart was included on the back of the form in both English and metric measurements for an easy, quick reference so that a calculator would not be needed. There was also a precalculated column for classifying patients $<80 \%$ of ideal body weight. The tool's section on weight history began with the initial question of whether or not there was any planned weight loss. If the patient responded positively, then an attempt was made to determine the time frame in which the weight loss took place. The percentage of weight loss was then calculated and compared with a table that collated the length of time to the percent of weight loss. Weight loss of $1 \%$ to $5 \%$ within 1 month before admission and $7 \%$ to $10 \%$ in the preceding months was considered to be a nutritional risk.

B. Testing for reproducibility. Registered nurses on an adult general care unit were instructed on the administration of the screening tool through inservices. During this training period, two patient cases were reviewed for each of the two classifications. Patients were excluded from the study if they were comatose or disoriented or if there was no family member or significant other to answer the questions on the screening form. After completion of the screening tool, nurses classified the patient as at nutritional risk or low nutritional risk. A nutritionist also completed the screen independently of nursing and classified each patient for nutritional risk. All admission screens were completed within 48 hours of admission.

Phase 2: Validating the Nutrition Screening Tool. Phase 2 of the study was conducted to validate the screening tool. In this phase of the investigation, the participant's prealbumin concentrations were measured. Exclusion criteria included patients with disorders known to affect prealbumin, excluding malnutrition. These disorders included the following: diabetes, liver disease, renal disease, hyperthyroidism, presence of an acute phase response, and active, chronic inflammatory diseases. ${ }^{21}$ Other exclusion criteria included patients $<18$ years of age, patients who were comatose or disoriented and for whom a family member or significant other was not present to answer the nutrition screening questions. After instruction and case review, a registered nurse without formal prior nutritional training completed the nutrition screen within 24 hours of admission. After completion of the screen the patient was classified as at nutritional risk or low nutritional risk.

Prealbumin samples were obtained from the admission blood sample. Low serum prealbumin was considered to be a value $<16 \mathrm{mg} / \mathrm{dL}$. All prealbumins were measured by rate nephelometry using the ARRAY 360 System (Beckman Instruments, Inc, Brea, CA).

Statistics. To test the reproducibility of the tool, it was determined that 135 screens were needed to detect a $15 \%$ difference between the nurse and nutritionist with a significance level of .05 and a power of .80 . To test the validity of the tool, it was determined that 28 patients per classification would be required to detect a $30 \%$ difference with a significance level of .05 and a power of $.80 . A \chi^{2}$ goodness of fit test was used to determine the interobserver reliability. Specificity and sensitivity was used to measure the validity of the tool. A $p$ value of $<.05$ 
Table 1. Admission nutrition screening tool

A. Diagnosis

If the patient has at least $\mathrm{ONE}$ of the following diagnoses, circle and proceed to section $\mathrm{E}$ to consider the patient AT NUTRITIONAL RISK and stop here.

Anorexia nervosa/bulimia nervosa

Malabsorption (celiac sprue, ulcerative colitis, Crohn's disease, short bowel syndrome)

Multiple trauma (closed-head injury, penetrating trauma, multiple fractures)

Decubitus ulcers

Major gastrointestinal surgery within the past year

Cachexia (temporal wasting, muscle wasting, cancer, cardiac)

Coma

Diabetes

End-stage liver disease

End-stage renal disease

Nonhealing wounds

B. Nutrition intake history

If the patient has at least $\mathrm{ONE}$ of the following symptoms, circle and proceed to section $\mathrm{E}$ to consider the patient AT NUTRITIONAL RISK and stop here.

Diarrhea $(>500 \mathrm{~mL} \times 2$ days)

Vomiting ( $>5$ days)

Reduced intake $(<1 / 2$ normal intake for $>5$ days)

C. Ideal body weight standards

Compare the patient's current weight for height to the ideal body weight chart on the back of this form.

If at $<80 \%$ of ideal body weight, proceed to section $\mathrm{E}$ to consider the patient AT NUTRITIONAL

RISK and stop here.

D. Weight history

Any recent unplanned weight loss? No

If yes, within the past

Yes months

Current weight (lbs or $\mathrm{kg}$ )

Usual weight (lbs or $\mathrm{kg}$ )

Height ( $\mathrm{ft}$, in or $\mathrm{cm}$ )

Find percentage of weight lost: usual wt - current wt $\times 100=$ Amount (lbs or kg) weeks or

Compare the \% wt loss with the chart values and circle appropriate value

\begin{tabular}{lcc} 
Length of time & Significant $(\%)$ & Severe $(\%)$ \\
\hline 1 week & $1-2$ & $>2$ \\
2-3 weeks & $2-3$ & $>3$ \\
1 month & $4-5$ & $>5$ \\
3 months & $7-8$ & $>8$ \\
5 + months & 10 & $>10$
\end{tabular}

If the patient has experienced a significant or severe weight loss, proceed to section $\mathrm{E}$ and consider the patient AT NUTRITIONAL RISK

E. Nurse assessment

Using the above criteria, what is this patient's nutritional risk? (circle one) LOW NUTRITIONAL RISK AT NUTRITIONAL RISK

was considered significant. Values are expressed as means $\pm S D$.

\section{RESULTS}

Phase 1: Testing the Reproducibility of the Nutrition Screening Tool. A total of 186 patients were screened during the first phase of the study. The age range was from 14 to 78 years of age (mean $45.7 \pm$ 18.1 years). Ninety-two men $(49.7 \%$ ) and 94 women $(50.5 \%)$ entered the study. The mean age for men was $43.1 \pm 18$ years and the mean age for women was 48.2 \pm 18 years. Both the nurse and the nutritionist assigned the identical nutrition classification in 181 of the 186 patients (Table 2). Eighty-three patients (44.6\%) were classified as at nutritional risk by both 
the nutritionist and the nurse, and 98 patients $(52.7 \%)$ were classified as at low nutritional risk by the two disciplines. Of the five remaining patients, two $(1.1 \%)$ were classified as low nutritional risk by the nurse and at nutritional risk by the nutritionist and three $(1.6 \%)$ were classified as at risk by the nurse and as low risk by the nutritionist. Thus there was $97.3 \%$ agreement between the two raters, which was not significant $\left(\chi^{2}=.047, p=.95\right)$.

Phase 2: Validating the Nutrition Screening Tool. During phase 2 of the study, screening was completed on 57 patients. One participant was excluded because of an insufficient quantity of serum to obtain a PAB result. Twenty-eight participants (50\%) were women and $28(50 \%)$ were men. The mean age was $56.9 \pm 16.3$ years. Twenty-nine patients $(50.9 \%)$ were classified at low nutritional risk (mean age $58.7 \pm 15.7$ years) and $27(49.1 \%)$ as at nutritional risk (mean age $55.2 \pm 16.9$ years). Of the 29 patients who were classified as at nutritional risk, $13(44.8 \%)$ were entered because of diagnosis, $10(34.5 \%)$ reported symptoms related to nutrition intake history, $1(3.4 \%$ ) was less than ideal body weight, and 5 (17.2\%) experienced unplanned weight loss prior to admission. Of the 29 patients at low nutritional risk, 27 had normal admission PAB and 2 had low PAB (Table 3). Of the 27 patients classified as at risk, 16 had normal PAB and 11 had low PAB. These results were significant, $\chi^{2}=8.9, p<.003$. The sensitivity of the tool was determined to be $84.6 \%$; the specificity was $62.7 \%$. The positive predictive value or the proportion of those with a low prealbumin concentration who were malnourished was $40.7 \%$ and the negative predictive value or the proportion of those with a normal prealbumin concentration who were not malnourished was $93.1 \%$.

\section{DISCUSSION}

The purpose of developing a screening tool is to identify people at risk early so that treatment can be started before complications occur. ${ }^{22}$ The ideal screen should be inexpensive and easy to administer and should impose minimal discomfort to the patient. Additionally, the tool must be valid, reliable, and

Table 2. Agreement of patient nutritional risk by interobservers*

\begin{tabular}{lrrr}
\hline & \multicolumn{2}{c}{ Classification by nurse } & Total \\
\cline { 2 - 3 } & At risk & Low risk & \\
\hline Classification by nutritionist & & & \\
At nutritional risk & 83 & 2 & 85 \\
Low nutritional risk & 3 & 98 & 101 \\
Total & 86 & 100 & 186 \\
\hline
\end{tabular}

*Not significant $\left(\chi^{2}=.047, p=.95\right)$. reproducible. Screening does not diagnose illness but identifies those who test positive and who will require further evaluation. In this case, the patient at risk would be referred to a dietitian who would perform a complete assessment including adequacy of nutrient intake (current, previous, and required), nutritional implications of selected laboratory tests or their results, conditions that may affect gastrointestinal function, physical examination for manifestation of nutrient deficiency or excess, food intolerance or allergies, and food preferences and diet prescriptions.

The screening tool used in this investigation included nutrition parameters that had previously been demonstrated to be robust by Baker et al in $1982 .{ }^{15}$ Their tool required health care professionals to complete an objective nutrition assessment on admission based on physical exam and history and then to classify patients as either well nourished, moderately malnourished, or severely malnourished. In their study, the SGA was compared with standard nutrition parameters that included anthropometric evaluation, percentage of ideal weight, percentage of ideal lean body weight, percentage of body fat, creatinine-height index, serum albumin, serum transferrin, total lymphocyte count, total-body nitrogen, total-body potassium, and delayed cutaneous hypersensitivity to four antigens. Analysis of variance demonstrated no statistical differences in total lymphocyte count, serum transferrin, and total-body nitrogen. A follow-up study completed in 1984 concluded that serum albumin, serum transferrin, anthropometry, and the creatinine-height index reduced sensitivities and specificities for predicting nutrition-associated complications when compared to SGA. ${ }^{23} A$ third study, conducted by these same investigators, using a larger sample size and two hospitals, found transferrin, creatinine-height index, percent ideal weight, percent body fat, and total lymphocyte count were not useful in predicting complications. ${ }^{24}$ Mean values of weight loss, percent of weight loss, and duration of dietary change increased significantly among the three nutrition categories with the highest values occurring in the severely malnourished. A study by Hirsch et al ${ }^{18}$

Table 3. Classification of participant according to prealbumin level and nutritional risk*

\begin{tabular}{lrcc}
\hline & \multicolumn{2}{c}{ Prealbumin } & Total \\
\cline { 2 - 3 } & Low & Normal & \\
\hline Nutrition classification & & & \\
At nutritional risk & 11 & 16 & 27 \\
Low nutritional risk & 2 & 27 & 29 \\
Total & 13 & 43 & 56 \\
\hline
\end{tabular}

* Significant $\left(\chi^{2}=8.9, p \leq .003\right)$. 
used SGA on a medical-surgical gastroenterology service found that weight loss and the underlying illness were the variables that influenced practitioners on the final nutrition classification. Our screening tool was based on the results of these studies with emphasize on diagnosis, nutrition history, physical exam, and ideal body weight.

The reproducibility of the SGA has been tested in other studies and confirms our findings of high inter-rater agreement between nutrition classification. In the original study, two raters who were physicians agreed on the exact clinical classification in 48 of 59 patients or $81 \%{ }^{15}$ In the follow-up study in two teaching hospitals by a group of five clinicians ( 3 physicians who were residents in clinical nutrition, 1 research nurse, and 1 nurse practitioner), there was $91 \%$ agreement in the SGA ratings. ${ }^{17}$ Other studies involving health professionals cited agreements of $79 \%$ and $80 \%$ between the two observers. ${ }^{18,18}$

Validity of the SGA in the previously cited studies was accomplished by stratifying patients who were at high risk for nutrition-associated complications. These complications included evidence of a wound or deep sepsis (ie, pneumonia or abdominal abscess) or positive blood cultures, the use of antibiotics, and length of stay. The authors found the incidence of infection significantly increased with the severity of the nutrition classification, as did the length of stay and the use of antibiotics. To test the validity of our screening tool, we choose PAB as our "gold standard." This is the best biochemical indicator available to detect depletion of visceral protein in selected patient populations. To allow valid interpretation of PAB levels, we excluded patients with disorders known to effect PAB levels. Unlike albumin, which has a half-life of 19 to 20 days, and transferrin, which has a half-life of 8 to 10 days, PAB has a short half-life of 2 to 3 days with substantially smaller body pools. ${ }^{21}$ Because of the long half-lives of albumin and transferrin, changes will occur too slowly to reflect rapid changes in nutritional status. Therefore, PAB levels allow the clinician to determine which patients would most likely benefit from early intensive nutrition evaluation and intervention before complications occur. Patients with diabetes, renal or liver failure, hyperthyroidism, presence of an acute phase response, and active chronic inflammatory disease were not included in phase 2 of our study because of the nonnutritional influence these conditions have on prealbumin concentrations (our chosen measurement standard). In many of these conditions, the patient would be classified as "at nutritional risk" in step 1 of the screening tool. Unfortunately, there is no biochemical marker that is reflective of nutritional status for patients with active inflammation. We are not able to tell from our study how well these individuals would be classified for their nutritional status by our screen.
The sensitivity (the proportion of patients with low prealbumins that were classified as "at nutritional risk") of our screening tool was $84.6 \%$ and the specificity (the proportion of patients with normal prealbumins that were classified as "no nutritional risk") was $62.7 \%$. An ideal screening tool is one that is $100 \%$ sensitive and $100 \%$ specific; however, generally this is not possible. An improvement in the specificity of our screen could have been achieved by using a more stringent definition of nutritional risk. This could have been done by increasing the number of days patients experienced diarrhea, vomiting, or reduced intakes by increasing the amount of weight loss required to be classified as "at risk." This would have reduced the number of patients misclassified as "at risk," reducing the "false negatives." However, changing the definition would have increased the number of patients with low prealbumin classified as "no risk," increasing the number of "false positives" and thereby reducing the sensitivity. In our investigation the relative importance of correctly classifying all patients possibly at nutritional risk outweighed the impact of misclassifying those not at nutritional risk. The consequences of the high sensitivity and low specificity of our screen was that more patients would have been referred to a dietitian for a complete nutrition assessment than would have been necessary. This alternative was more desirable because it does not allow malnourished patients to slip through the screening process undetected.

To our knowledge, this is the first study to describe the use of a valid reproducible tool for early identification of nutritional risk by nurses. Expanding nutrition awareness into the scope of multidiscipline practitioners embodies the spirit of the recent JCAHO revisions. This "Agenda for Change" recommends that nutrition care and other patient care services be seen as multidisciplinary processes that integrate several departments and qualified individuals as appropriate. Further studies in smaller, non-tertiary care settings are needed to determine the applicability of this tool in those patient populations. The need for a nutrition screen relies on the premise that early detection of malnutrition will result in reduced costs from shorter, less complicated hospitalization. A feasibility study is required to determine the costs versus the benefits of implementing this screening tool.

\section{REFERENCES}

1. Weinsier RL, Hunker EM, Krumdieck CL, et al. A prospective evaluation of general medical patients during the course of hospitalization. Am J Clin Nutr 1979;32:418-26.

2. Mullen JL, Buzby GP, Matthews DC, et al. Reduction of operative morbidity and mortality by combined preoperative and postoperative nutritional support. Ann Surg 1980; 192:604-13.

3. Reilly JJ, Hull SF, Albert N, et al. Economic impact of malnutrition: a model system for hospitalized patients. JPEN 
$1988 ; 12: 371-6$

4. McClave SA, Mitoraj TE, Thielmeier KA, et al. Differentiating subtypes (hypoalbuminemic vs marasmic) of protein-calorie malnutrition: incidence and clinical significance in aniversity hospital setting. JPEN 1992;16:337-42.

5. Kushner RF, Ayello EA, Beyer PL, et al. National coordinating committee clinical indicators of nutrition care. J Am Diet Assoc 1994;94:1168-77.

6. Foltz MB, Schiller MR, Ryan AS. Nutrition screening and assessment: current practices and dietitians leadership role. J Am Diet Assoc 1993;93:1388-95.

7. Hedberg A, Garcia N, Trejus TJ, et al. Nutritional risk screening: development of a standardized protocol using dietetic technicians. J Am Diet Assoc 1988;88:1553-6.

8. Hunt DR, Maslovitz A, Rowlands BJ, et al. A simple nutrition screening procedure for hospital patients. J Am Diet As8oc 1985;85:332-6.

9. Kamath SK, Lawler M, Smith AE, et al. Hospital malnutrition: a 33 hospital screening study. J Am Diet Assoc 1986;86:203-6.

10. Nagel MR. Nutrition screening: identifying patients at risk for malnutrition. NCP 1993;8:171-6.

11. Sayarath VG. Nutrition screening for malnutrition: potential economic impact at a community hospital. J Am Diet Assoc 1993;93:1440-2.

12. Seltzer MH, Bastidas JA, Cooper DM, et al. Instant nutritional assessment. JPEN 1979;3:157-9.

13. Thompson JS, Burrough CA, Green JL, et al. Nutritional screening in surgical patients. J Am Diet Assoc 1984;84:337-8.

14. Christensen KS, Gstundtner KM. Hospital-wide screening improves basis for nutrition intervention. J Am Diet Assoc
$1985 \cdot 85: 704-6$

15. Baker JP, Detsky AS, Wesson DE, et al. Nutritional assessment: a comparison of clinical judgment and objective measurements. New Engl J Med 1982;306:969-72.

16. Detsky AS, Smalley PS, Chang J. Is this patient malnourished? JAMA 1994;271:54-8.

17. Detsky AS, McLaughlin JR, Baker JP, et al. What is subjective global assessment of nutritional status? JPEN 1987;11:8-13.

18. Hirsch $S$, de Obaldia $N$, Petermann $M$, et al. Subjective global assessment of nutritional status: further validation. Nutrition 1991;7:35-8.

19. Hasse J, Strong S, Gorman M, et al. Subjective global assessment: alternative nutrition-assessment technique for liver-transplant candidates. Nutrition 1993;9:339-43.

20. Enia G, Sicuso C, Alati G, et al. Subjective global assesement of nutrition in dialysis patients. Nephrol Dial Transplant 1993;8:1094-8.

21. Heymsfield SB, Tighe A, Wang Z. Nutritional assessment by anthropometric and biochemical methods. In: Shils ME Olson JA, Shike M, eds. Modern nutrition in health and disease, 2nd ed. Philadelphia: Lea \& Febiger, 1994;812-41.

22. Hennekens CH, Buring JE. Screening. In: Mayrent SL, ed. Epidemiology in medicine. Boston: Little, Brown and Com. pany, 1987;327-47.

23. Detsky AS, Baker JP, Mendelson RA, et al. Evaluating the accuracy of nutritional assessment techniques applied to hospitalized patients: methodology and comparisons. JPEN 1984;8:153-9.

24. Detsky AS, Baker JP, O'Rourke K, et al. Predicting nutrition-associated complications for patients undergoing gastrointestinal surgery. JPEN 1987;11:440-6.

\section{American Society for Parenteral and Enteral Nutrition}

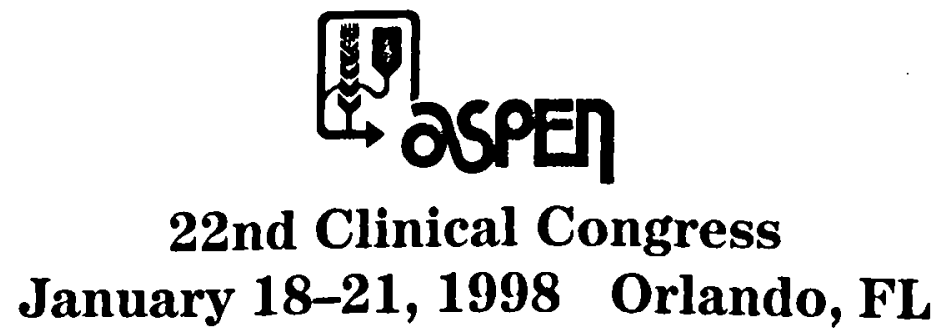

A.S.P.E.N.'s Abstract Review Committee is calling for scientists engaged in basic or clinical research relative to nutrition and metabolic support to submit their work for presentation at the upcoming Clinical Congress. There are three formats for presentation of scientific studies: podium presentation, poster presentation, and the new scientific information exchange format. Abstract forms will appear in the May 1997 issue of the Journal of Parenteral and Enteral Nutrition(JPEN), or you may call A.S.P.E.N. and request that you be mailed abstract forms. Deadline for receipt of abstracts is August 22, 1997.

Direct inquiries to:

A.S.P.E.N., 8630 Fenton Street, Suite 412, Silver Spring, MD 20910

Phone (301) 587-6315 or Fax (301) 587-2365

e-mail aspen@access.digex.net 